EDITORIAL

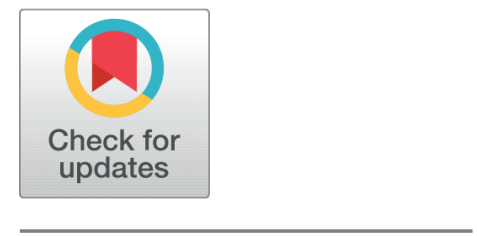

open AcCess

Received: 22.12 .2021

Accepted: 02.01.2022

Published: 12.01 .2022

Citation: Patil M. (2021). Hypertension and Nutrition Core Group of IAPEN India. Journal of Nutrition Research. 9(1): 1-3. https:// doi.org/10.55289/jnutres/v9i1.09

* Corresponding author.

drpatilmansi@gmail.com

Funding: None

Competing Interests: None

Copyright: ( $) 2021$ Patil. This is an open access article distributed under the terms of the Creative Commons Attribution License, which permits unrestricted use, distribution, and reproduction in any medium, provided the original author and source are credited.

Published By India Association for Parenteral and Enteral Nutrition (IAPEN)

ISSN

Electronic: 2348-1064

\title{
Hypertension and Nutrition Core Group of IAPEN India
}

\author{
Mansi Patil ${ }^{1 *}$ \\ 1 Asha Kiran JHC Hospital, Chinchwad, Pune, India
}

\section{Abstract}

The rising incidence of hypertension in the developing countries is an indication of changing lifestyles and an epidemiological transition with a shift from communicable to non-communicable diseases. Hypertension often goes undiagnosed and is frequently diagnosed accidentally or when there are complications. Raised blood pressure is a long-standing condition and needs lifestyle changes for both prevention and management. The Hypertension and Nutrition Core Group of IAPEN India aims at training the healthcare professionals and the community towards preventing, diagnosing and managing hypertension and following healthy lifestyles. It is imperative that the healthcare professionals be trained to encourage task sharing and task shifting in order to manage the rising burden of hypertension.

Keywords: Hypertension; Hypertension and Nutrition Core Group of IAPEN India; Lifestyle management in hypertension; Task sharing; Task shifting; diagnostic inertia in hypertension

\section{Introduction}

In emerging nations, the burden of noncommunicable diseases (NCDs) is growing. NCDs have a complex aetiology, thus it's critical to assess the association between various risk factors and these lifestyle illnesses. Hypertension is responsible for $31 \%$ of all worldwide deaths, with poor and medium income nations accounting for $1 / 4$ of the total. Furthermore, only $37 \%$ of people with hypertension in India have their blood pressure under control, a figure that jumps to $57 \%$ with pharmacologic intervention ${ }^{(1,2)}$. As a result, primary hypertension prevention would have significant public health ramifications.
Overweight and obesity, physical inactivity, and a poor diet are a few of the modifiable risk factors for hypertension that have been documented. Excessive alcohol use and abstinence have recently been recognised as separate, modifiable risk factors for the development of hypertension in women ${ }^{(3)}$. Although the impact of treatments to change one or more of these variables have been proven, the percentage of new onset hypertension might be avoided by changing a combination of lifestyle factors. Among the various modalities, Blood pressure (BP) measurement accuracy is critical for hypertension diagnosis and therapy. 


\section{Aims and Vision}

The Hypertension and Nutrition Core Group, has a vision to reduce the modifiable risk factors of hypertension and diagnose the burden of undiagnosed hypertension by $50 \%$ in the next 10 years and improve the awareness of hypertension and its risk factors among both the community and healthcare professionals (HCPs). To achieve this vision, the hypertension and Nutrition Core group aims to disseminate information in the community, train health care providers to overcome diagnostic inertia and identify undiagnosed hypertension to achieve better control.

\section{Need for Hypertension Management}

In the past 3 decades the rate of mortality due to Hypertension in Asia has doubled ${ }^{(3)}$. Asia being a diverse continent with the largest population burden in the world, consists of heterogeneous ethnicity and income groups, majority of them falling under upper middle to low middle income groups ${ }^{(4)}$. Highest hypertension mortality rate in Asia was observed in Turkmenistan and Kazakhstan (400 per 100,000 population). Hypertension rates varied amongst Asian countries, but pooled mainly at central and eastern Asian countries than those in South-eastern, Eastern, and Western countries ${ }^{(3)}$. In a study by World Health Statistics 2020 that provided agestandardized prevalence of hypertension in adults in the Asian countries, prevalence was more than $20 \%$ in 39 of $47(83 \%)$ and was more than $25 \%$ in 23 of 47 (49\%) Asian countries $^{(3)}$. India, Nepal, Pakistan, have been evidenced to be in the early stage of the Hypertension epidemic, whereas countries like Turkmenistan, Kazakhstan and Uzbekistan have been included amongst the stage 2 - rapidly increasing hypertension epidemic ${ }^{(3)}$. Risk factors, particularly tobacco/ alcohol consumption, high salt intake along with poor diet quality and overweight/obesity have a major contribution for incidences of hypertension in Central and Southern Asian countries $^{(2)}$.

The CARRS study found varying awareness and control rates of hypertension in the South Asian region with the highest awareness levels among women in Karachi at $57 \%$ and lowest among men in Delhi at 22\%. Involvement and training of the community health workers could be an effective lowcost scale-up strategy to improve hypertension control in the South and Central Asian regions ${ }^{(5)}$.

The hypertension core group has actively participated in national and international awareness campaigns and conferences.

\section{Need for training HCPs}

Task shifting and task sharing are two ways proposed by the World Health Organization (WHO) to increase and ensure access to critical health services by maximising the utilisation of the current healthcare workforce. Task shifting and task sharing allow tasks to be shifted from highly trained healthcare workers (HCWs) to HCWs with less training and qualifications, respectively, and jobs to be shared with an equally competent cadre of HCWs. During the COVID19 pandemic, this enables for more efficient use of limited human resources working in overcrowded health systems ${ }^{(6)}$.

Task sharing is also a deliberate action that involves a group of health professionals working together in a team to complete a task or provide a service that they haven't done previously. Unlike informal and haphazard task shifting/sharing, planned techniques include training, certification, and support. Task shifting is the name given to a process of delegation in which responsibilities are delegated to less skilled health personnel when suitable. Task shifting can make more efficient use of the human resources already available by rearranging the workforce in this way ${ }^{(7)}$.

Multitasking or task switching helps a person to quickly and effectively adapt to a variety of situations. When you switch tasks, you make more mistakes than when you execute one activity at a time. These time and mistake penalties rise as the activities get more complicated. Each task switch may just take a fraction of a second, but if you perform a lot of switching in a day, it can cost you $40 \%$ of your productivity ${ }^{(7)}$.

These two techniques might be part of a bigger plan to enhance and strengthen health-care system capacity in order to increase access to hypertension treatment while sustaining important health-care services. Furthermore, by lowering the strain of the healthcare profession, these techniques can assist safeguard and sustain their health and well-being ${ }^{(7)}$.

There is broad agreement that boosting human resources for health should be seen as a top priority, and that Task Shifting should be a key component of any endeavour to ensure universal access to hypertension prevention, treatment, and care. It was also acknowledged that Task Shifting is a considerable departure from traditional health-care approaches and, as such, has the potential to fundamentally alter the health-care delivery system. Increased access, wider coverage, and enhanced quality of care will arise from addressing some of the underlying structural constraints for widespread service delivery. Furthermore, the Task Shifting strategy will assist to the improvement of primary health care services and systems by permitting decentralisation of services to underserved regions. Task Shifting is a long-term approach for strengthening health systems, not just a quick fix for hypertension $^{(8)}$.

There has been considerable apprehension that the Task Shifting approach might result in non-professional health workers replacing well-trained health professionals. Task Shifting is not intended to be used in this way, according to experience. Task shifting entails a more sensible division of labour and shared duties across the various cadres of health professionals in order to increase the efficiency of 
the present workforce ${ }^{(9)}$. By outsourcing suitable activities to community health workers and other lay providers, Task Shifting can grow the total pool of human resources for health swiftly. Task shifting will not eliminate the need to recruit, train, and retain a larger number of health workers at all levels, including senior professional cadres. The Task Shifting suggestions will assist in determining the necessary number of employees required for efficient hypertension care delivery and will suggest methods in which those numbers might be increased.

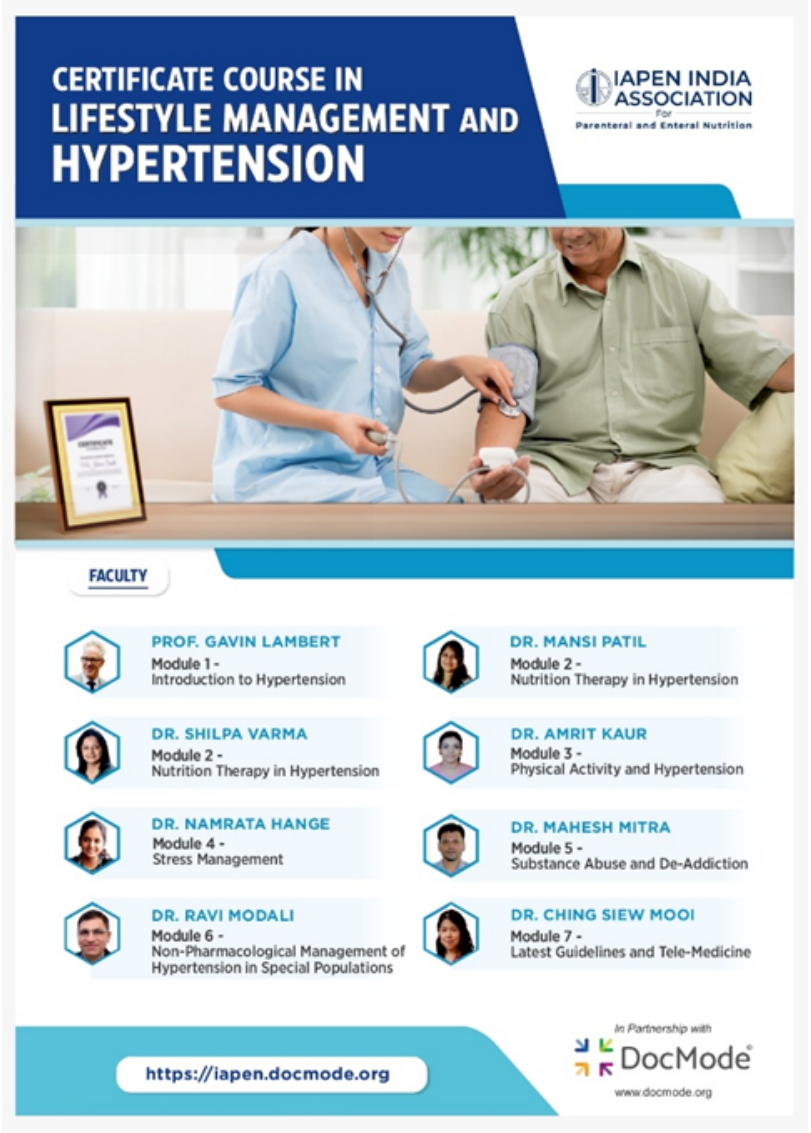

\section{Activities by the Hypertension and Nutrition Core Group}

The Hypertension and Nutrition Core group has been a part of various national and international campaigns and has been one of the leaders of the May Measurement Month (MMM) Campaign for measuring blood pressure in the community. MMM is an international community based blood pressure campaign initiated by the International Society of Hypertension and the World Hypertension League. The Hypertension and Nutrition Core Group has screened over 200,000 individuals and published the findings in peer reviewed indexed journals. The core group has also spearheaded the training for healthcare professionals towards primary prevention of hypertension by designing a course on Lifestyle Management in Hypertension which is a unique course encompassing nutritional management, designing an exercise regime, stress management, alcohol and substance de-addiction as a well as management of hypertension in special populations. The course lays emphasis on empowering healthcare professionals to encourage task shifting and task sharing.

\section{Conclusion}

With the growing incidence of hypertension globally, preventive strategies play an important role in the prevention of hypertension. It is often noted, that correct and effective guidance is important in the right transfer of knowledge to the healthcare professionals as well as the community.

\section{References}

1) Schutte AE, Venkateshmurthy NS, Mohan S, Prabhakaran D. Hypertension in Low- and Middle-Income Countries. Circulation Research. 2021;128(7):808-826. Available from: https://dx.doi.org/10.1161/ circresaha.120.318729.

2) Aringazina A, Kuandikov T, Arkhipov V. Burden of the Cardiovascular Diseases in Central Asia. Central Asian Journal of Global Health. 2018;7(1):321. Available from: https://dx.doi.org/10.5195/cajgh.2018. 321.

3) Zhao D. Epidemiological Features of Cardiovascular Disease in Asia. JACC: Asia. 2021;1(1):1-13. Available from: https://doi.org/10.1016/j. jacasi.2021.04.007.

4) Kario K, Chia YC, Sukonthasarn A, Turana Y, Shin J, Chen $\mathrm{CH}$, et al. Diversity of and initiatives for hypertension management in Asia-Why we need the HOPE Asia Network. The Journal of Clinical Hypertension. 2020;22(3):331-343. Available from: https://dx.doi.org/10.1111/jch. 13733.

5) Patel SA, Deepa M, Shivashankar R, Ali MK, Kapoor D, Gupta R, et al. Comparison of multiple obesity indices for cardiovascular disease risk classification in South Asian adults: The CARRS Study. PLOS ONE. 2017;12(4):e0174251. Available from: https://dx.doi.org/10.1371/ journal.pone.0174251.

6) Prabhakaran D, Jeemon P, Ghosh S, Shivashankar R, Ajay VS, Kondal $\mathrm{D}$, et al. Prevalence and incidence of hypertension: Results from a representative cohort of over 16,000 adults in three cities of South Asia. Indian Heart Journal. 2017;69(4):434-441. Available from: https://dx.doi. org/10.1016/j.ihj.2017.05.021.

7) Finkelstein EA, Krishnan A, Naheed A, Jehan I, de Silva HA, Gandhi $\mathrm{M}$, et al. Budget impact and cost-effectiveness analyses of the COBRABPS multicomponent hypertension management programme in rural. The Lancet Global Health. 2021;9(5):e660-e667. Available from: https: //doi.org/10.1016/S22147109X(21)00033-4.

8) https://www.hope-asia-network.com/ . 2022.

9) Beaney T, Schutte AE, Stergiou GS, Borghi C, Burger D, Charchar F, et al. May Measurement Month 2019; The Global Blood Pressure Screening Campaign of the International Society of Hypertension. Hypetension. 2020;2020(76):333-341. Available from: https://doi.org/10. 1161/HYPERTENSIONAHA.120.14874. 